Plane-Wave Detection:

A Nonlinearly Ill-Posed

Inverse Problem

William W. Symes

September, 1989

TR89-12 



\title{
Plane-Wave Detection: a Nonlinearly Ill-Posed Inverse Problem
}

\author{
William W. Symes \\ Department of Mathematical Sciences \\ Rice University \\ Houston, Texas 77005 U.S.A.
}

September 1989

\begin{abstract}
Acknowledgements. It is a pleasure indeed to thank Professor Gary Roach for his magnificent hospitality during the Workshop on Inverse Problems and Imaging, Ross Priory, 16-21 July 1989; for the opportunity to present this material there; and for the invitation to publish it in these Proceedings. Professor Guy Chavent of Université de Paris IX (Dauphine) and the Program CEREMADE are also due thanks for their support of the research reported here, which was also partially supported by Grant No. N00014-J-89-1119 and the National Science Foundation under grant DMS 86-03614.
\end{abstract}





\section{Introduction}

It is commonplace that inverse problems of applied mathematics are often illposed. Such a problem generally takes the (idealized) form of a functional equation involving a map $\phi$, representing the physical connection between model and data

$$
\phi(x)=z
$$

with $x$ (the model, or solution) and $z$ (the data) ranging over suitable function spaces, on which the map $\phi$ is defined. Such a problem is said to be ill-posed when-roughly speaking-- the solution $x$ does not depend continuously on the data $z$. A common, and much-studied, source of ill-conditioning is the appearance of arbitrary small singular values of the linearized map $D \phi(x)$. We might call the instability of the solution resulting from small singular values of $D \phi$ linear ill-posedness. See e.g. Tikonov and Arsenin (1974).

Some inverse problems exhibit a form of effective ill-posedness of quite different nature. This second pathology may appear when the functional equation is re-written as a best-fit problem:

$$
\min _{x}\|\phi(x)-z\|
$$

with a suitable choice of norm \|\| . Such reformulation might be motivated 
by the recognition that the range of $\phi$ does not contain all interesting data points $z$-i.e. that the data is likely to be inconsistent, perhaps because of simplifications in the physics used to formulate the map $\phi$. If $\phi$ is "sufficiently noninear," the cost function

$$
J(x ; z)=\|\phi(x)-z\|
$$

may be nonconvex, with many local minima over a prescribed set of admissible models $x$. For various reasons--e.g. computational cost of global search-one may be forced to regard any local minimum of $J$ as a "solution." Then the existence of many (local) minima must be regarded as a form of instability of the solution--i.e. nonlinear ill-posedness.

One inverse problem exhibiting a severe case of nonlinear ill-posedness is the velocity estimation problem of reflection seismology. For an extensive discussion of this rather specialized problem, with many references, we direct the reader to the monograph by Santosa and the author (Santosa and Symes, 1989). In the following pages we will mostly discuss instead a much simpler problem, the plane wave detection problem, which shares the essential mathematical features of the velocity estimation problem without carrying the conceptual baggage of reflection seismology. In the final section we will de- 
scribe briefly a version of velocity estimation, to make plausible this sharing of features.

A deep understanding of nonlinear ill-posedness and related matters is to be had through G. Chavent's theory of quasiconvex sets in Hilbert space (Chavent, 1980). The plane-wave detection problem is treated from the point of view of Chavent's theory in Symes (1989). In the present paper we describe just the basic properties of a simple best-fit formulation of the detection problem $(\S 2)$, and then indicate how the cost function can be convexified and smoothed (§3). The fourth section begins by describing the acoustic model of reflection seismology, then presents several simplifications and approximations leading to a problem recognizably similar to plane wave detection. We give a brief discussion of this problem, referring the interested reader to other papers for more information. 


\section{The Output Least-Squares Formulation}

We suppose that the function $z(\xi, t)$ is a sampling on $\{\eta=0\}$ of the threedimensional field $U(\xi, \eta, t)$ :

$$
z(\xi, t)=U(\xi, 0, t), \quad-1 \leq \xi \leq 1, \quad t \in \mathbb{R}
$$

We also suppose that $U$ is (approximately) a plane-wave moving at speed 1 , i.e.

$$
U(\xi, \eta, t)=u(t-\xi \sin \theta-\eta \cos \theta)+n(\xi, \eta, t)
$$

for a waveform $u(t)$, an angle $\theta$, and a noise component $n(\xi, \eta, t)$. The planewave detection problem is:

Given $z(\xi, t)$, estimate the waveform $u(t)$ and the angle $\theta$ (or equivalently the sine $s=\sin \theta$ ).

Because one believes that the noise $n(\xi, 0, t)$ is small in the mean-square sense, or for statistical reasons (Tarantola, 1987), one may assume that $z \in$ $L^{2}([-1,1] \times \mathbb{R})$, and ask that the data $z$ be predicted optimally in the leastsquares sense by a pair $u(t), s$. That is, making also the a priori assumption 
that supp $u \subset[0,1]$, i.e. $u \in L^{2}[0,1]$, we define

$$
\begin{aligned}
& \phi[s, u](\xi, t)=u(t-s \xi) \\
& \phi:[-1,1] \times L^{2}[0,1] \rightarrow L^{2}([-1,1] \times \mathbb{R})
\end{aligned}
$$

Set

$$
\begin{aligned}
E_{0} & =[-1,1] \times L^{2}[0,1] \\
F & =L^{2}([-1,1] \times \mathbb{R})
\end{aligned}
$$

with obvious norms. Then an output-least squares formulation of the planewave detection problem is:

$$
\min _{x \in E_{0}}\|\phi[x]-z\|_{F}^{2}
$$

Remark One could imagine that $U$ represents the far-field signal of an underwater acoustic source. Then the problem becomes that of estimating the waveform emitted by the source and its direction relative to a line of receivers. With this interpretation, our problem becomes a caricature of an important problem in ocean acoustics. We note that our point of view is quite different from that taken in the ocean acoustics literature.

An obstacle to the study of the above least-squares problem is immediately evident: As defined, the map $\phi$ is

- continuous, but 
- nowhere locally uniformly continuous,

- hence $a$ fortiori nowhere differentiable.

In fact

$$
\phi[s+\delta s, u](\xi, t)-\phi[s, u](\xi, t)=u(t-(s+\delta s) \xi)-u(t-s \xi)
$$

and the stated properties of $\phi$ follow from familiar properties of the shift on $L^{2}(\mathbb{R})$. Thus we cannot study the dependence of the solution on the data via the implicit function theorem or related tools, nor can we use Newton's method or its relatives to compute minima with any confidence of convergence.

The map $\phi$ becomes of class $C^{2}$ if its domain is restricted, say, to

$$
E_{2}:=[-1,1] \times H_{0}^{2}[0,1]
$$

This restriction does not cure the problem of its delinquent features, of course. To begin with, in any $F$-neighborhood of any consistent data point $z=\phi(x)$, there exist consistent data $z^{\prime}=\phi\left(x^{\prime}\right)$, with $\left\|x^{\prime}\right\|_{E_{2}}$ as large as one likes. Therefore some regularization of the optimization problem is necessary, in order that the solution depend stably on the data, even though $\phi$ is not a 
smoothing operator! This is a rather trivial sort of instability, however; the actual state of affairs is much worse. We will establish:

There exist consistent data $z=\phi\left(x_{0}\right)$ with $\left\|x_{0}\right\|_{E_{2}} \leq 1$ for which the problem

$$
\min _{x:\|x\|_{E_{2} \leq 1}}\|\phi(x)-z\|_{F}
$$

has at least two (local) solutions.

Thus restricting the $\mathrm{H}^{2}$-size of the solution does not restore well-posedness to the best-fit version of the detection problem, even for noise-free data!

Set $z(\xi, t)=u_{0}(t)=a \chi(t) \sin \omega t$ with $\omega$ and $a$ to be determined, and $\chi \in C_{0}^{\infty}(0,1)$ fixed. Then

$$
z=\phi\left[x_{0}\right], \quad x_{0}=\left[0, u_{0}\right]
$$

For $u \in L^{2}[0,1], s \in[-1,1], x=[s, u]$,

$$
\begin{aligned}
& \langle z, \phi[x]\rangle=a \int_{-1}^{1} d \xi \int d t u(t-s \xi) \chi(t) \sin \omega t \\
& =a \int_{-1}^{1} d \xi \int d t u(t) \chi(t+s \xi)(\sin \omega t \cos \omega s \xi+\cos \omega t \sin \omega s \xi) \\
& =2 a \frac{\sin \omega s}{\omega s} \int d t u(t) \chi(t) \sin \omega t \\
& \quad+\int_{-1}^{1} d \xi \int d t u(t)(\chi(t+s \xi)-\chi(t))[\sin \omega t \cos \omega s \chi+\omega \cos \omega t \sin \omega s \xi]
\end{aligned}
$$


There is a uniform estimate for derivatives of $\chi$ of order $\leq 2$ :

$$
\left|\chi^{(k)}(t+s \xi)-\chi^{(k)}(t)\right| \leq C|s|
$$

for $s \in[-1,1], \xi \in[-1,1], t \in \mathbb{R}$ and $k \leq 2$. Accordingly an integrationby-parts argument shows that the second term is bounded in absolute value by

$$
a \omega^{-2} C|s|\|u\|_{H^{2}[0,1]} .
$$

Thus

$$
\langle z, \phi[x]\rangle_{F}=2 \frac{\sin \omega s}{\omega s}\left\langle u, u_{0}\right\rangle_{L^{2}[0,1]}+O\left(\omega^{-2} a|s|\|u\|_{H^{2}[0,1]}\right)
$$

So

$$
\begin{aligned}
\|\phi[x]-z\|_{F}^{2}= & \|\phi[x]\|_{F}^{2}+\|z\|_{F}^{2}-2\langle\phi[x], z\rangle_{F} \\
\geq & 2\left[\left\|u_{0}\right\|_{L^{2}[0,1]}^{2}+\|u\|_{L^{2}[0,1]}^{2}-2 \frac{\sin \omega s}{\omega s}\left\langle u, u_{0}\right\rangle_{L^{2}[0,1]}\right] \\
& -C \omega^{-2} a|s|\|u\|_{H^{2}[0,1]} \\
\geq & s\left[\left(1-\left|\frac{\sin \omega s}{\omega s}\right|\right)\left(\|u\|_{L^{2}[0,1]}^{2}+\left\|u_{0}\right\|_{L^{2}[0,1]}^{2}\right]\right) \\
& -C \omega^{-2} a|s|\|u\|_{H^{2}[0,1]}
\end{aligned}
$$

Integration-by-parts shows that there exist $C_{1}, C_{2}>0$ so that

$$
a^{2}\left(C_{1}+\frac{C_{2}}{\omega}\right) \geq\left\|u_{0}\right\|_{L^{2}[0,1]}^{2} \geq a^{2}\left(C_{1}-\frac{C_{2}}{\omega}\right) .
$$


The hypotheses of the statement to be proved allow us to require that $\|u\|_{H^{2}[0,1]} \leq 1$. Thus for $s_{0}=\frac{\pi}{\omega}$ and $x=\left[s_{0}, u\right]$ we get

$$
\begin{aligned}
\|\phi[x]-z\|_{F} & \geq 2 a^{2}\left(C_{1}-\frac{C_{2}}{\omega}\right)-C a \pi \omega^{-3} \\
& \geq a^{2} C_{1}(2-\epsilon)
\end{aligned}
$$

provided that

$$
C_{1} \epsilon \geq \frac{2 C_{2}}{\omega}+\frac{C \pi}{a \omega^{3}}
$$

Now $\left\|u_{o}\right\|_{H^{2}[0,1]} \leq 1$, provided $|\omega| \geq 1$ and $a \omega^{2} \leq K$ for suitable $K>0$. Thus we take $a=K \omega^{-2}$ so that the above condition becomes

$$
C_{1} \epsilon \geq\left(2 C_{2}+C \pi K^{-1}\right) \omega^{-1}
$$

and is satisfied for any choice of $\epsilon>0$ as soon as $\omega$ is large enough.

Now consider the special choice $u=\alpha u_{0}$. Then

$$
\|\phi[x]-z\| \leq 2\left[1+\alpha^{2}-2 \alpha \frac{\sin \omega s}{\omega s}\right]\left\|u_{0}\right\|_{L^{2}[0,1]}^{2}+C a \omega^{-2}|s| \alpha\left\|u_{0}\right\|_{H^{2}[0,1]}
$$

Choose $s_{1}=\frac{5 \pi}{2 \omega}, x=\left[s_{1}, \alpha u_{0}\right]$; then the above is

$$
\begin{aligned}
& \leq 2\left[1+\alpha^{2}-\frac{4 \alpha}{5 \pi}\right]\left\|u_{0}\right\|_{L^{2}[0,1]}^{2}+3 C a \alpha \pi \omega^{-3}\left\|u_{0}\right\|_{H^{2}[0,1]} \\
& \leq 2 a^{2}\left[1+\alpha^{2}-\frac{4 \alpha}{5 \pi}\right]\left(C_{1}+\frac{C_{2}}{\omega}\right)+3 a C \alpha \pi \omega^{-3} .
\end{aligned}
$$


Choose $\alpha=\frac{2}{5 \pi}$; we get

$$
\begin{aligned}
& =2 a^{2}\left[1-\frac{4}{(5 \pi)^{2}}\right]\left(C_{1}+\frac{C_{2}}{\omega}\right)+a \frac{6 C}{5 \omega^{3}} \\
& =a^{2} C_{1}\left[2\left(1-\frac{4}{(5 \pi)^{2}}\right)+2\left(1-\frac{4}{5 \pi^{2}}\right) \frac{C_{2}}{C_{1} \omega}+\frac{6}{5} \frac{C}{C_{1} K \omega}\right] \\
& \leq a^{2} C_{1}\left(2-\frac{4}{(5 \pi)^{2}}\right)
\end{aligned}
$$

for $\omega$ large enough.

Now choose $\epsilon=(5 \pi)^{-2}$. Then we have shown that, for $\omega$ sufficiently large

(i) for any $u$ with $\|u\|_{H^{2}[0,1]} \leq 1, \quad s_{0}=\frac{\pi}{\omega}, \quad x=\left[s_{0}, u\right]$ :

$$
\|\phi[x]-z\|_{F} \geq a^{2} C_{1}(2-\epsilon)
$$

(ii) for $s_{1}=\frac{5 \pi}{2 \omega}, \quad u_{1}=\frac{2}{5 \pi} u_{0}, x_{1}=\left[s_{1}, u\right]$

$$
\left\|\phi\left[x_{1}\right]-z\right\|_{F} \leq a^{2} C_{1}(2-2 \epsilon)
$$

Since any continuous path from $x_{1}$ to $x_{0}$ must pass over the set $\{x=[s, u]:=$ $\frac{\pi}{\omega}$, we have shown that the set

$$
\left\{x=[s, u]:\|\phi[x]-z\|_{F} \leq a^{2} C_{1}(2-3 / 2 \epsilon)\right\}
$$

is not connected; in particular the component containing $x_{1}$ is disjoint from the component containing $x_{0}$. The connected component of $x_{0}$ is contained 
in

$$
C_{0}=\left\{x=[s, u]:|s| \leq \frac{\pi}{\omega}-\delta,\|u\|_{H^{2}[0,1]} \leq 1\right\}
$$

for a suitable choice of $\delta>0$, and the connected component of $x_{1}$ is contained in

$$
C_{1}=\left\{x=[s, u]: \frac{\pi}{\omega}+\delta \leq s \leq 1,\|u\|_{H^{2}[0,1]} \leq 1\right\}
$$

which follows from the uniform continuity of $\phi$ on $[-1,1] \times H^{\mathbf{1}}[0,1]$ and the compactness of the injection $H^{2} \rightarrow H^{1}$. The sets $C_{0}$ and $C_{1}$ are closed, bounded, and convex in $E_{2}$, hence weakly closed, whence follows the existence of a local minimizer in each. In particular, we have established the existence of a local minimum distinct from $x_{0}$, as required. q.e.d.

It is easy to extend this reasoning to generate examples with any number of local minima whatsoever. Thus even the restriction of $\phi$ to a ball in $E_{2}$ does not suffice to render the output least-squares problem well-posed in the nonlinear sense. 


\section{The Coherence Reformulation}

To motivate the next step, regard the data $z(\xi, t)$ of the detection problem as a number of independent time series measurements, parameterized by the location $\xi$ along the $x$-axis. This point of view reflects accurately the way such measurements are actually made - in reality, only a "few" receivers may be deployed, and the sample rate in $\xi$ is far lower than that in $t$.

The plane-wave hypothesis implies that these time series are not independent, but are tied rigidly together by the time delay rate $s$. The difficulty described in the last section is also a consequence of this rigidity. It is simply very difficult to match all of the time series at once with any but precisely the "right" time delay rate. Any other choice results in large mismatch somewhere.

Our solution to the quandary of the hyperactive behaviour of the model is to relax it. We allow independent models of the various traces, constrained by a penalty for deviation from the plane-wave hypothesis. The penalty is not "capital punishment," as in the least-squares approach; models inconsistent with the plane-wave hypothesis are permitted, but required to pay a "fine" related to their deviation. The "fine schedule," ie. penalty weight, is a very 
important determinant of efficiency of such a scheme.

Precisely, we introduce a cover $\tilde{\phi}$ of the map $\phi$, that is a diagram of maps and spaces

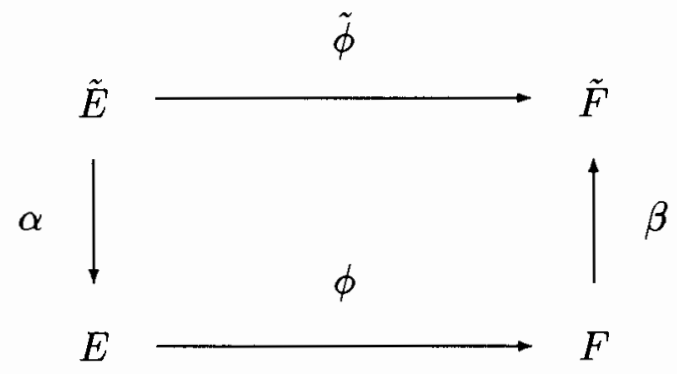

which partially commutes:

$$
\text { if } \begin{aligned}
\tilde{\phi}(\tilde{x}) & \in \mathcal{R}(\beta), \text { then } \\
\tilde{\phi}(\tilde{x}) & =\beta(\phi(\alpha(\tilde{x}))) .
\end{aligned}
$$

We also assume that $\beta$ is injective. As we shall see, it is possible for such a partially commuting diagram to be constructed with a differentiable $\tilde{\phi}$, even though $\phi$ is not differentiable.

First note the consequence: if

$$
\tilde{z}=\beta(z)
$$

and $\tilde{\phi}(\tilde{x})=\tilde{z}$, then $x=\alpha(\tilde{x})$ satisfies $\phi(x)=z$, since $\beta$ is injective. That is, the functional equation for $\tilde{\phi}$ has the "same" solutions as the functional 
equation for $\phi$, provided that the data for the former is the image under $\beta$ of the data for the latter.

The space $\tilde{E}$, as a set, is

$$
\begin{aligned}
\tilde{E} & =[-1,1] \times\left\{\tilde{u} \in H^{1}([-1,1] \times[-1,3]):\right. \\
\tilde{u}(\xi, t) & \equiv 0, \quad t<-|\xi| \\
\frac{\partial \tilde{u}}{\partial t}(\xi, t) & \equiv 0, \quad t>1+|\xi|\} .
\end{aligned}
$$

We shall actually need a family of Hilbert spaces $\tilde{E}_{\sigma}, \sigma>0$, consisting of the set $\tilde{E}$ equipped with the norm

$$
\|[s, \tilde{u}]\|_{\tilde{E}, \sigma}^{2}=\left\|\frac{\partial \tilde{u}}{\partial t}\right\|_{0}^{2}+\sigma^{2}\left(|s|^{2}+\left\|\frac{\partial \tilde{u}}{\partial \xi}\right\|_{0}^{2}\right)
$$

(here the subscript " 0 " denotes, as before, the norm in $L^{2}([-1,1] \times[-1,3])$.)

We shall also need a family of maps

$$
\tilde{\phi}_{\sigma}: \tilde{E}_{\sigma} \rightarrow \tilde{F}:=F \oplus F
$$

defined by

$$
\tilde{\phi}_{\sigma}[s, \tilde{u}]=\left(\begin{array}{c}
\frac{\partial \tilde{u}}{\partial t} \\
\sigma\left(\frac{\partial \tilde{u}}{\partial \xi}+s \frac{\partial \tilde{u}}{\partial t}\right)
\end{array}\right) .
$$

Finally, $\alpha$ and $\beta$ are given by

$$
\alpha[s, \tilde{u}]=\left[s, \frac{1}{2} \int_{-1}^{1} d \xi \frac{\partial \tilde{u}}{\partial t}(t-s \xi)\right]
$$




$$
\beta[f]=\left(\begin{array}{l}
f \\
0
\end{array}\right) .
$$

Obviously $\beta$ is injective. Also, if $\tilde{\phi}[s, \tilde{u}] \in \mathcal{R}(\beta)$, then

$$
\frac{\partial \tilde{u}}{\partial \xi}-s \frac{\partial \tilde{u}}{\partial t}=0
$$

so $\frac{\partial \tilde{u}}{\partial t}(\xi, t)=u(t+s \xi)$ for some $u \in L^{2}(\mathbb{R})$.

Because of the assumptions about the support of $\tilde{u}$, supp $u \subset[0,1]$.

Moreover

$$
\alpha[s, \tilde{u}]=[s, u] .
$$

Necessarily

$$
\tilde{\phi}[x, \tilde{u}]=\beta(\phi[s, u]) .
$$

As noted above, for consistent data $\tilde{z} \in \mathcal{R}(\beta)$, the functional equation for $\tilde{\phi}$ has the "same" solution as that for $\phi$. On the other hand, $\tilde{\phi}_{\sigma}: \tilde{E}_{\sigma} \rightarrow \tilde{F}$ is differentiable - even real-analytic. Of course the diagram above could not commute if all maps but $\phi$ were differentiable. In fact, $\alpha$ is not $C^{1}$. However, application of $\alpha$ may be viewed as a "postprocess," to get from $\tilde{\phi}^{-1}(\tilde{z})$ to $\phi^{-1}(z)$, and does not entail solving a functional equation.

Because $\tilde{\phi}_{\sigma}$ is $C^{2}$ or better, local well-posedness of the least-squares prob- 
lem

$$
\min _{(s, \tilde{u})}\left\|\tilde{\phi}_{\sigma}[s, \tilde{u}]-\tilde{z}\right\|^{2}=J_{\sigma}[s, \tilde{u} ; \tilde{z}]
$$

for near-consistent $z$ follows from a coercivity estimate on the derivative $D \tilde{\phi}$.

Thus assume that

$$
\tilde{\phi}_{\sigma}\left[s_{0}, \tilde{u}_{0}\right]=\tilde{z}_{0}=\beta\left(z_{0}\right)
$$

We shall prove that

$$
\|[\delta s, \delta \tilde{u}]\|_{\tilde{E}, \sigma} \leq L_{\sigma}\left[u_{0}\right]\left\|D \phi_{\sigma}\left[s_{0}, \tilde{u}\right][\delta s, \delta \tilde{u}]\right\|_{\tilde{F}}
$$

for some $L_{\sigma}>0$. It follows immediately from this estimate and the implicit function theorem that $J_{\sigma}$ has a unique minimum near $\left[s_{0}, \tilde{u}_{0}\right]$ for $\tilde{z}$ near $\tilde{z}_{0}$.

The main step in the proof of the coercivity estimate is the estimate

$$
\left\|\frac{\partial \tilde{u}_{0}}{\partial t}\right\|_{0}^{2}|\delta s|^{2} \leq K_{0}\left\|\frac{\partial \delta \tilde{u}}{\partial t}\right\|_{0}^{2}+K_{1}\left\|D W\left[s_{0}, \tilde{u}_{0}\right][\delta s, \delta \tilde{u}]\right\|_{0}^{2}
$$

with $K_{0}, K_{1}$ independent of $\left[s_{0}, \tilde{u}_{0}\right]$. Here $W$ is the second component of $\phi_{1}$ :

$$
W[s, \tilde{u}]=\frac{\partial \tilde{u}}{\partial \xi}-s \frac{\partial \tilde{u}}{\partial t}
$$

and it is assumed that $\tilde{\phi}\left[s_{0}, \tilde{u}_{0}\right] \in \mathcal{R}(\beta)$, i.e. in particular that $W\left[s_{0}, \tilde{u}_{0}\right] \equiv 0$. Set

$$
\begin{aligned}
D: & =D W\left[s_{0}, \tilde{u}_{0}\right][\delta s, \delta \tilde{u}] \\
& =-\frac{\partial \tilde{u}_{0}}{\partial t} \delta s-\frac{\partial \delta \tilde{u}}{\partial t} s_{0}+\frac{\partial \delta \tilde{u}}{\partial \xi}
\end{aligned}
$$


Pick $\xi_{1}, \xi_{2} \in[-1,1], t_{2}=t_{1}+s_{0}\left(\xi_{2}-\xi_{1}\right)$. Then integrate both sides of the previous equation along the line segment

$$
\xi \in\left[\xi_{1}, \xi_{2}\right] \mapsto\left(\xi, t_{1}+s_{0}\left(\xi-\xi_{1}\right)\right)
$$

to get

$$
\begin{aligned}
\frac{\partial \tilde{u}_{0}}{\partial t}\left(\xi_{1}, t_{1}\right) \delta s\left(\xi_{2}-\xi_{1}\right)= & \int_{\xi_{1}}^{\xi_{2}} d \xi D\left(\xi_{1}, t_{1}+s_{0}\left(\xi-\xi_{1}\right)\right)+ \\
& +\delta \tilde{u}\left(\xi_{1}, t_{1}\right)-\delta \tilde{u}\left(\xi_{2}, t_{1}+s_{0}\left(\xi_{2}-\xi_{1}\right)\right) .
\end{aligned}
$$

According to the definition of $\tilde{E}, \delta \tilde{u} \equiv 0$ for $t<|\xi|$, so $(t \leq 3)$

$$
|\delta \tilde{u}(\xi, t)|^{2} \leq 4 \int_{-1}^{3} d t^{\prime}\left|\frac{\partial \delta \tilde{u}}{\partial t}\left(\xi, t^{\prime}\right)\right|^{2}
$$

so

$$
\begin{aligned}
\left(\xi_{2}-\xi_{1}\right)^{2}|\delta s|^{2}\left|\frac{\partial \tilde{u}_{0}}{\partial t}\left(\xi_{1}, t_{1}\right)\right|^{2} \leq & 3 \int_{\xi_{1}}^{\xi_{2}} d \xi\left|D\left(\xi, t_{1}+s\left(\xi-\xi_{1}\right)\right)\right|^{2}+ \\
& +12 \int_{-1}^{3} d t\left[\left|\frac{\partial \delta \tilde{u}}{\partial t}\left(\xi_{1}, t\right)\right|^{2}+\left|\frac{\partial \delta \tilde{u}}{\partial t}\left(\xi_{2}, 5\right)\right|^{2}\right] .
\end{aligned}
$$

Now integrate both sides in $\xi_{2}, t_{1}$ and $\xi_{1}$ in that order to get

$$
\frac{2}{3}|\delta s|^{2}\left\|\frac{\partial \tilde{u}_{0}}{\partial t}\right\|_{0}^{2} \leq 12\|D\|_{0}^{2}+48\left\|\frac{\partial \delta \tilde{u}}{\partial t}\right\|_{0}^{2}
$$

whence we can take $K_{0}=72, K_{1}=18$. Thus the "main step" estimate is established. 
To finish the proof of the coercivity estimate, note that

$$
\left\|\frac{\partial \delta \tilde{u}}{\partial \xi}\right\|_{0}^{2} \leq 3\left[\|D\|_{0}^{2}+|\delta s|^{2}\left\|\frac{\partial \tilde{u}_{0}}{\partial t}\right\|_{0}^{2}+\left|s_{0}\right|^{2}\left\|\frac{\partial \delta \tilde{u}}{\partial t}\right\|_{0}^{2}\right]
$$

whence

$$
\begin{aligned}
& \left\|\frac{\partial \tilde{u}_{0}}{\partial t}\right\|_{0}^{2}\|[\delta s, \delta \tilde{u}]\|_{\tilde{E}, \sigma}^{2}= \\
& =\left\|\frac{\partial \tilde{u}_{0}}{\partial t}\right\|_{0}^{2}\left[\left\|\frac{\partial \delta \tilde{u}}{\partial t}\right\|_{0}^{2}+\sigma^{2}\left(|\delta s|^{2}+\left\|\frac{\partial \delta \tilde{u}}{\partial \xi}\right\|_{0}^{2}\right)\right] \\
& \leq\left(\left\|\frac{\partial \tilde{u}_{0}}{\partial t}\right\|_{0}^{2}+72 \sigma^{2}+219 \sigma^{2}\left\|\frac{\partial u_{0}}{\partial t}\right\|^{2}\right)\left\|\frac{\partial \delta \tilde{u}}{\partial t}\right\|_{0}^{2}+\sigma^{2}\left(18+57\left\|\frac{\partial u_{0}}{\partial t}\right\|^{2}\right)\|D\|_{0}^{2} .
\end{aligned}
$$

Thus the coercivity estimate is proved with

$$
L_{\sigma}\left[s_{0}, \tilde{u}_{0}\right]=\max \left\{1+219 \sigma^{2}+72 \sigma^{2}\left\|\frac{\partial \tilde{u}_{0}}{\partial t}\right\|^{-2}, \sigma^{2}\left(57+18\left\|\frac{\partial \tilde{u}_{0}}{\partial t}\right\|^{-2}\right)\right\} .
$$

As mentioned above, this estimate ensures the local existence, uniqueness, and continuous dependence of the global minimizer of $J_{\sigma}$ for near-consistent data, i.e. data near the intersection of the range of $\phi_{\sigma}$ and the range of $\beta$. This local well-posedness result is just the beginning of the story, of course. Two questions of immediate "practical" interest are:

(i) How does one ensure that an initial estimate of $\tilde{x}=[s, \tilde{u}]$ lies sufficiently close to the global minimum of $J_{\sigma}$ to permit convergence of a Newton-like iterative scheme? 
(ii) How can one be sure that the data is, or is not, close enough to consistent data that the local well-posedness result holds?

The first question is addressed in the paper (Symes, 1989) using Chavent's quasiconvexity theory. There we show that a suitable initial estimate, and so a convergent quasi-Newton sequence, may be constructed, provided that

(a) the noise level is sufficiently low;

(b) $\sigma$ is chosen sufficiently small initially, and later increased to provide the maximum level of stability.

All of these results rely on estimates which are doubtless overly conservative. A major open problem is the derivation of algorithms to estimate appropriate values of $\sigma$ and of the noise level, hence answering question (ii). 


\section{The Seismic Reflection Inverse Problem}

In this section we give a rather terse description of the inverse problem of reflection seismology, and explain its relation to the plane wave detection problem discussed in the preceding sections.

Reflection seismology is an active remote sensing technique. It currently yields the most highly detailed images available for study of the earth's crust to depths of $15-30 \mathrm{~km}$. The method was developed by the petroleum industry, but enjoys some use in academic geophysics (tectonics, continental margins) and engineering geophysics (structure placement, groundwater) as well.

The data of reflection seismology are measurements of the transient response of the crust to controlled energy sources-explosive charges, compressed air devices, dropped weights, hydraulic vibrators, etc. The measurements of ground velocity (on land) or pressure (at sea) are made by a (typically linear) array of sensors. A typical marine seismic cable used in oil and gas exploration may be $3 \mathrm{~km}$. long and contain several hundred hydrophones (immersible microphones), wired together in groups to produce signals on 48,96 , or even more channels. The sample rate for recording these 
signals is $250-1000$ samples per second, and the typical digitally recorded signal may be six seconds long. The source may be repeatedly stimulated, each 10 seconds or so, as the exploration vessel steams along towing the cable. A brief calculation shows that the amount of data recorded in a day's work by such a ship is truly phenomenal. In fact, the geophysical exploration industry has been for many years the single largest industrial user of digital magnetic tape. An excellent and easily accessible overview of reflection seismology and other geophysical prospecting methods is provided by Dobrin (1975).

The extraction of useful information from this vast quantity of data is particularly challenging because of the complex physics of seismic wave propagation. In fact, this physics is only poorly understood. It is generally accepted that some modification of linear elasticity might be suitable, as the limited time-scale and bandwidth of seismic waves conspire to hide or average the microscopic mechanics of rocks. In fact most of the research literature, and almost all production software, is based on the acoustic model, which represents the earth by a fluid. The local linear response of a fluid is characterized by a density $\rho(x)$ and sound velocity $c(x)$, both functions of spatial position. If the energy source is assumed isotropic (it isn't), and small (on the scale of the dominant wavelength, say) and positioned at $x_{s}$ then the excess pressure 
$p(x, t)$ obeys

$$
\begin{gathered}
\frac{1}{\rho(x) c(x)^{2}} \frac{\partial^{2} p}{\partial t^{2}}(x, t)-\nabla \cdot \frac{1}{\rho(x)} \nabla p(x, t)=f(t) \delta\left(x-x_{s}\right) \\
p(x, t) \equiv 0, \quad t<<0 .
\end{gathered}
$$

In principle, a boundary condition is also needed at the earth/air interface $\left(x_{3}=0:\right.$ the earth's surface is flat on the scale of the exploration seismic experiment!), but for simplicity we shall assume that $\rho, c$ are constant, at fixed, known values for $x_{3}<0$ ("up" in geophysics!).

This initial value problem represents the simplest model of seimic wave propagation retaining most of its notable features. In terms of the model, the basic problem of reflection seismology is to recover the mechanical descriptors of the earth, i.e. $\rho(x)$ and $c(x)$, from the idealized surface seismogram

$$
s[\rho, c]\left(x_{1}, x_{2}, t\right)=p\left(x_{1}, x_{2}, 0, t\right) .
$$

To exhibit the close relation with the plane-wave detection problem, three further simplifications are useful. Each limits the utility of the model further - though the end result can still be used successfully to process field seismic data!

First, we assume "the earth is layered," i.e. that $\rho$ and $c$ depend only on $z=x_{3}$. This assumption is a reasonable approximation as sediments which 
later become rock are laid down in horizontal layers, and retain a large degree of lateral homogeneity in many areas. Because of the assumed translation invariance, we can replace the seismogram by a transform in the horizontal variables. We use the Radon transform, suitably scaled:

$$
P(\xi, z, t)=\iint d x_{1} d x_{2} p\left(x_{1}, x_{2}, z, t+\xi x_{1}\right)
$$

which obeys

$$
\begin{gathered}
\frac{1}{\rho(z)}\left(\frac{1}{c^{2}(z)}-\xi^{2}\right) \frac{\partial^{2} P}{\partial t^{2}}(\xi, z, t)-\frac{\partial}{\partial z} \frac{1}{\rho(z)} \frac{\partial}{\partial z} P(\xi, z, t)=f(t) \delta\left(z-z_{s}\right)=0 \\
P(\xi, z, t)=0, \quad t<<0 .
\end{gathered}
$$

The plane-wave seismogram is

$$
S[\rho, c](\xi, t)=P(\xi, 0, t) .
$$

Note that the I.V.P. for $P$ is hyperbolic where $|\xi| c(z)<1$.

The second simplification is based on the recognition that the density and velocity in the sedimentary earth exhibit a dichotomy of scales: small-scale fluctuation in the meters-tens of meters range, corresponding to seasonal and short-term changes in weather patterns and depositional environment, and long scale fluctuations in the hundreds or thousands of meters corresponding to geological, epochal changes. It was recognized early that the short-scale 
fluctuations could be viewed usefully as perturbations about the long-scale trends (though the mathematical justification for this linearization is recent and still partial). Accordingly write

$$
\begin{aligned}
& \rho=\rho_{s}+\rho_{r} \\
& c=c_{s}+c_{r}
\end{aligned}
$$

where the subscripts " $s$ " and " $r$ " stand for "smooth" and "rough" respectively. Regular perturbation about $c_{s}, \rho_{s}$ gives

$$
D S\left[\rho_{s} ; c_{s}\right]\left[\rho_{r}, c_{r}\right]=\left.\delta P\right|_{z=0}
$$

where

$$
\frac{1}{\rho_{s}}\left(\frac{1}{c_{s}}-\xi^{2}\right) \frac{\partial^{2} \delta P}{\partial t^{2}}-\frac{\partial}{\partial z} \frac{1}{\rho_{s}} \frac{\partial \delta P}{\partial z}=\frac{1}{\rho_{s}} \frac{2 c_{r}}{c_{s}^{3}} \frac{\partial^{2} P}{\partial t^{2}}-\frac{\partial}{\partial z} \frac{\rho_{r}}{\rho_{s}^{2}} \frac{\partial P}{\partial z}
$$

and

$$
\delta P \equiv 0, \quad t<<0
$$

Note that $\delta P$ is linear in $\rho_{r}$ and $c_{r}$, but nonlinear in $\rho_{s}$ and $c_{s}$.

The third simplification is to use geometric optics to approximate the solution of the above perturbational boundary value problem. This step is justified by the recognition that the wavelengths measured in seismic records are quite short compared to scale of the slowly-varying coefficients $\left(c_{s}\right.$ and 
$\left.\rho_{s}\right)$. After some work (e.g. Santosa and Symes, 1988) one obtains

$$
D S\left[\rho_{s}, c_{s}\right]\left[\rho_{r}, c_{r}\right](\xi, t) \approx A\left(\rho_{s}, c_{s} ; \xi, t\right)\left[\frac{\rho_{r}}{\rho_{s}}+\frac{c_{r}}{c_{s}}+c_{s}^{2} \xi^{2} \frac{\rho_{r}}{\rho_{s}}\right] \circ \zeta(\xi, t)
$$

Here $\zeta$ is the inverse two-way traveltime, defined implicitly by

$$
t=2 \int_{0}^{\zeta(\xi, t)} d z \sqrt{\frac{1}{c_{s}^{2}(z)}-\xi^{2}}
$$

and $A\left(\rho_{s}, c_{s} ; \xi, t\right)$ is an overall amplitude factor, determined by ray geometry.

Close examination of the last two equations reveals the following comparisons with the plane-wave detection problem:

(1) The Radon parameter ("slowness") $\xi$ plays the role of receiver coordinate $\xi ; t$ is time in both cases.

(2) The smooth background velocity $c_{s}$ plays the role of the direction sine $s$, in that it determines a $\xi$-dependent change of variables: for the reflection seismology problem,

$$
t \mapsto \zeta(t, \xi) ;
$$

for the plane-wave detection problem, the time shift $t \mapsto t+s \xi$.

(3) $\rho_{r} / \rho_{s}$ and $c_{r} / c_{s}$ play the role of the plane waveform $u(t)$. 
In a number of recent papers, we have made deductions for the reflection seismology model similar to those sketched in sections $1-3$ for the planewave detection problem. We have shown that the map $\left(\rho_{s}, c_{s}, \rho_{r}, c_{r}\right) \mapsto$ $D S\left[\rho_{s}, c_{s}\right]\left[\rho_{r}, c_{r}\right]$ is either non-differentiable or has non-coercive derivative, depending on choice of metric in the domain. We have also posed a coherency method, by replacing

$$
\begin{aligned}
\frac{\rho_{r}(z)}{\rho_{s}(z)}+\frac{c_{r}(z)}{c_{s}(z)} & \mapsto r_{z}(t, \xi) \\
\frac{\rho_{r}(z)}{\rho_{s}(z)} & \mapsto r_{d}(t, \xi)
\end{aligned}
$$

and requiring that the reflectivities $r_{i}$ and $r_{d}$ satisfy the coherency conditions:

$$
\begin{aligned}
& \frac{\partial}{\partial \xi} r_{i} \circ \zeta=0 \\
& \frac{\partial}{\partial \xi} r_{d} \circ \zeta=0
\end{aligned}
$$

These are analogous to the condition

$$
\left(\frac{\partial}{\partial \xi}+s \frac{\partial}{\partial t}\right) U(\xi, t) \equiv 0
$$

of the plane-wave detection problem. The fit-to-data and coherency constraints can be combined into a least-squares problem, just as was shown in Section 3. We have built a Fortran code to solve the resulting least-squares problem. 
The theory of this approach to the reflection seismology problem, including well-posedness estimates, extension to the original acoustic model (rather than the simplified, linearized plane wave model), and preliminary numerical experiments, are reported in Symes (1988a,b). Application of the coherency technique to field seismic data is accomplished in Symes and Carazzone (1989). 


\section{References}

1. CHAVENT, G. [1988]. Quasiconvex sets and size* curvature condition, application to nonlinear inversion, Rapport INRIA No. 1017.

2. DOBRIN, M. [1976]. Introduction to Geophysical Prospecting, 3rd edition, McGraw-Hill, New York.

3. SANTOSA, F. and W. SYMES [1988]. High frequency perturbational analysis for the point-source response of a layered acoustic medium, $J$. Comp. Phys. 74, pp. 318-381.

4. SANTOSA, F. and W. SYMES [1989]. An Analysis of Least-Squares Velocity Inversion, Geophysical Monograph No. 4, Society of Exploration Geophysicists, Tulsa.

5. SYMES, W. [1988a]. Velocity inversion by coherency optimization, Technical Report 88-4, Department of Mathematical Sciences, Rice University, Houston, TX 77251.

6. SYMES, W. [1988b]. Velocity inversion: a model problem from reflection seismology, Technical Report 88-13, Department of Mathematical Sciences, Rice University, Houston, TX 77251.

7. SYMES, W. [1989]. The plane-wave detection problem, preprint.

8. SYMES, W. and J. CARAZZONE, [1989]. Velocity inversion by coherency optimization, Technical Report 89-9, Department of Mathematical Sciences, Rice University, Houston TX 77251. 